\title{
MEDEX 2015
}

Sunderland, Angus; Freer, Joseph; Evans, Laura; Dolci, Alberto; Crotti, Matteo; Macdonald, Jamie

\section{High Altitude Medicine and Biology}

DOI:

10.1089/ham.2016.0145

Published: 01/09/2017

Peer reviewed version

Cyswllt i'r cyhoeddiad / Link to publication

Dyfyniad o'r fersiwn a gyhoeddwyd / Citation for published version (APA):

Sunderland, A., Freer, J., Evans, L., Dolci, A., Crotti, M., \& Macdonald, J. (2017). MEDEX 2015: Heart Rate Variability Predicts Development of Acute Mountain Sickness. High Altitude Medicine and Biology, 18(3), 199-208. https://doi.org/10.1089/ham.2016.0145

\footnotetext{
Hawliau Cyffredinol / General rights

Copyright and moral rights for the publications made accessible in the public portal are retained by the authors and/or other copyright owners and it is a condition of accessing publications that users recognise and abide by the legal requirements associated with these rights.

- Users may download and print one copy of any publication from the public portal for the purpose of private study or research.

- You may not further distribute the material or use it for any profit-making activity or commercial gain

- You may freely distribute the URL identifying the publication in the public portal ?
}

Take down policy

If you believe that this document breaches copyright please contact us providing details, and we will remove access to the work immediately and investigate your claim. 


\section{MEDEX 2015: Heart rate variability predicts development of}

\section{acute mountain sickness}

Angus Sutherland ${ }^{1}$ Joseph Freer ${ }^{2}$, Laura Evans ${ }^{3}$, Alberto Dolci ${ }^{4}$, Matteo Crotti ${ }^{5}$, Jamie Hugo Macdonald ${ }^{6}$

1. Anaesthetic Department, Portsmouth Hospitals Trust, Queen Alexandra Hospital. Mail to: Email: angus.sutherland@doctors.org.uk Tel: +44 (0) 7903366181

2. The BMJ, BMA House, Tavistock Square, London, WC1H9JP. Email: josephbfreer@gmail.com Tel: $+44(0) 7770905289$

3. Intensive Care Department, Northern General Hospital. Email: laura.evans@doctors.org.uk Tel: +44 (0) 7585563847

4. Faculty of Science and Technology, Cavendish Building, University of Westminster, 115 New Cavendish Street, London W1W 6UW. Email: a.dolci@westminster.ac.uk Tel: +44 (0) 7402875132.

5. School of Exercise and Sports Sciences, Università degli Studi di Milano, Milan, Italy. Email: crotti.mc@gmail.com Tel: +393202318944

6. Extremes Research Group, School of Sport, Health and Exercise Sciences, Bangor University, George Building, Bangor, Gwynedd, LL57 2PZ. Email: j.h.macdonald@bangor.ac.uk Tel: +44 (0) 1248383272

Corresponding author: Dr Angus Sutherland, Anaesthetic Department, Portsmouth Hospitals Trust, Queen Alexandra Hospital.

Word count -text: 4,712 (excluding abstract, tables, figures and references); -abstract: 249 Running title: Heart rate variability and AMS 


\section{Abstract:}

Aims: Acute mountain sickness (AMS) develops when the body fails to acclimatize to atmospheric changes at altitude. Pre-ascent prediction of susceptibility to AMS would be a useful tool to prevent subsequent harm. Changes to peripheral oxygen saturation $\left(\mathrm{SpO}_{2}\right)$ on hypoxic exposure have previously been shown to be of poor predictive value. Heart rate variability (HRV) has shown promise in the early prediction of AMS, but its use pre-expedition has not previously been investigated. We aimed to determine whether pre- and intra-expedition HRV assessment could predict susceptibility to AMS at high altitude with better diagnostic accuracy than $\mathrm{SpO}_{2}$.

Methods: 44 healthy volunteers undertook an expedition in the Nepali Himalaya to $>5000 \mathrm{~m} . \mathrm{SpO}_{2}$ and HRV parameters were recorded at rest in normoxia and in a normobaric hypoxic chamber prior to the expedition. The same data were later collected at various altitudes on the expedition along with daily Lake Louis Score to assess AMS symptomology.

Results: Low frequency/high frequency ratio (LF/HF) in normoxia and LF following 15 minutes of exposure to normobaric hypoxia had moderate (area under the curve $\geq 0.8$ ) diagnostic accuracy. LF/HF ratio in normoxia had the highest sensitivity $(85 \%)$ and specificity (88\%) for predicting AMS on subsequent ascent to altitude. In contrast pre-expedition $\mathrm{SpO}_{2}$ measurements had poor (area under the curve $<0.7)$ diagnostic accuracy and inferior sensitivity and specificity.

Conclusions: Pre-ascent measurement of HRV in normoxia has potentially better diagnostic accuracy for AMS prediction than all measures of $\mathrm{HRV}$ in hypoxia, and better than peripheral $\mathrm{SpO}_{2}$ saturation monitoring.

Keywords: AMS, altitude, expedition, hypoxia, peripheral oxygen saturation, Heart rate variability. 


\section{Introduction:}

Acute mountain sickness (AMS) is a complex syndrome presenting at altitudes above $2500 \mathrm{~m}$, and will affect approximately fifty percent of people above 4000m altitude (Maggiorini et al., 1990). It is characterised by headache, gastrointestinal symptoms, fatigue, insomnia, and neurological symptoms diagnosed clinically using the Lake Louise Score (Honigman et al., 1993; Lake Louise Consensus on the Definition and Quantification of Altitude Illness, 1991). Accurate prediction of AMS susceptibility is of importance to enable enhanced prevention measures via increased observation, behavioural changes (e.g. slower ascent profile) and/or prophylactic medication. However, a widelyaccepted method for prediction of AMS currently does not exist.

The exact mechanism causing AMS is unknown, but is thought to relate to neurohumoral or haemodynamic processes within the central nervous system (Hackett, 1999). Reduced arterial oxygen saturation is presumably the ultimate upstream cause and is known to be associated with increased reporting of AMS symptoms (Oliver et al., 2012). Nevertheless, participants with similar peripheral oxygen saturation $\left(\mathrm{SpO}_{2}\right)$ may or may not present with AMS (Luks and Swenson, 2011). As $\mathrm{SpO}_{2}$ decreases during ascent to high altitude, heart rate increases, presumably as a result of increased sympathetic activity and to maintain oxygen delivery (Brierley et al., 2012). Although the role of the autonomic nervous system in AMS is poorly understood it is conceivable that autonomic dysregulation could play an important role in its genesis (Duplain et al., 1999).

Despite the mechanism of AMS remaining elusive, many authors have attempted to develop tools to predict AMS susceptibility. The most commonly investigated tool remains $\mathrm{SpO}_{2}$ monitoring-Current evidence suggests that $\mathrm{SpOZ}$ values, determined 20-30 min after exposure to hypoxia equivalent to 2300-4200m, offer the most promise as useful predictors of AMS susceptibility (Burtscher et al., 2008). Unfortunately, such predictive tools have not shown high diagnostic accuracy and therefore have not been widely accepted (Burtscher et al., 2008, Luks and Swenson, 2011). Therefore, 
alternative or additional measures to improve AMS prediction are of interest. In this regard Burscher and colleagues demonstrated a small improvement in AMS prediction when combing $\mathrm{SpO}_{2}$ monitoring with the blood lactate response to hypoxia (Burtscher et al., 2008), but this invasive approach requires technical skills and is time-consuming. If autonomic dysregulation in response to hypoxia has a role in the genesis of AMS, then measures of peripheral autonomic activity such as heart rate variability (HRV), may provide an alternative additional predictive tool.

$\mathrm{HRV}$ is the beat-to-beat variation in the R-R intervals in an electrocardiogram and it has been suggested that it can be used to monitor the activity of the autonomic nervous system (Billman et al., 2011). The R-R interval data are analysed and the power of heart rate variability is quantified by determining the areas of the spectrum in different component widths: high frequency (HF; $0.15-0.4$ $\mathrm{Hz})$, low frequency $(\mathrm{LF} ; 0.04-0.15 \mathrm{~Hz})$, very low frequency $(\mathrm{VLF} ; \leq 0.04 \mathrm{~Hz}$ ) and ultra-low frequency (ULF; $\leq 0.0033 \mathrm{~Hz}$ ) (Camm et al., 1996; Task Force of the European Society of Cardiology and the North American Society of Pacing and Electrophysiology, 1996)

$\mathrm{HF}$ is widely accepted as an indicator of vagal tone. It is currently considered a good estimator of parasympathetic activation of the autonomic nervous system because the sympathetic nervous system accounts for only $10 \%$ of HF variability (Malliani et al., 1991, Billman, 2013). In contrast, it has been suggested that sympathetic and parasympathetic nervous system activation explains roughly $50 \%$ and $25 \%$ of the LF variability respectively (De Vito et al., 2002, Shaffer et al., 2014). In addition, LF currently is not considered a valid marker of autonomic nervous activity per se as it is influenced by the activity of baroreceptors in addition to those of the autonomic nervous system (Billman, 2013, De Vito et al., 2002, Rahman et al., 2011). A ratio of LF/HF has also been widely used as an indicator of sympatho-vagal balance. Taking into account what is known about LF, the LF/HF ratio may be more usefully described as a mutual relationship between baroreflex sensitivity and parasympathetic modulation (Rahman et al., 2011, Billman, 2013, Cohen and Taylor, 2002). Finally, a 
physiological explanation and the mechanism involved in the generation of VLF and ULF have not been clarified. Nevertheless, some authors suggest that circadian rhythms, core body temperature, metabolism, hormones, and intrinsic rhythms generated by the heart could be the cause of VLF and ULF fluctuations (Shaffer et al., 2014).

In clinical settings, HRV has been shown to be useful in post myocardial infarction risk stratification and in prediction of anxiety disorders (La Rovere et al., 1998, Chalmers et al., 2014). At altitude, a number of studies have investigated the dysregulation of the autonomic nervous system using HRV. These studies have shown a transient reduction in parasympathetic and increased sympathetic activity during acute exposure to hypobaric hypoxia (Kanai et al., 2001). However, there are few studies linking these alterations in the autonomic nervous system with AMS symptoms. Karinen and colleagues investigated the potential predictive value of HRV assessed during the actual ascent in determining later AMS susceptibility, showing good sensitivity and specificity in diagnosing AMS (Karinen et al., 2012), but if HRV is to become a useful applied tool in the prediction and management of AMS, its utility in assessment prior to expedition to altitude should be investigated. In this regard, Zhang et al. (2014) explored the response of the autonomic system when exposed to short term normobaric hypoxia and showed that even on acute short term exposure, a decrease in HRV is seen. Taken together the query remains of the possible predictive valve of HRV in normobaric hypoxia to predict the development of AMS symptoms on a subsequent expedition to altitude. Advances in heart rate telemetry monitoring systems have made it possible to evaluate HRV noninvasively and in a real-time manner. So, if successful, HRV may offer a low-cost, non-invasive tool to help identify those who are at risk of AMS prior to ascent (Burtscher et al., 2004).

The aim of this study was to determine whether HRV assessment prior to (acute normobaric normoxia or hypoxia) and during (chronic hypobaric hypoxia) an expedition, could predict susceptibility to AMS at high altitude with similar or better diagnostic accuracy to peripheral oxygen 
desaturation during acute normobaric hypoxia or chronic hypobaric hypoxia. We hypothesised that $\mathrm{LF}, \mathrm{HF}$ and their ratio assessed during 15 minutes of normobaric hypoxia $\left(\mathrm{FiO}_{2}=11.5 \%\right)$ would have acceptable and the best diagnostic accuracy to predict AMS susceptibility, defined as a Lake Louise Score of headache $\geq 1$ and total score $\geq 3$ during a subsequent trek to high altitude. 


\section{Materials and methods}

\section{Participants}

The study group consisted of 44 healthy participants (age range $24-62$ years; 26 male). Participant demographics are provided in table 1. Participants' maximum living altitude was $450 \mathrm{~m}$ and none of the participants had been above $3000 \mathrm{~m}$ during the month before the expedition. None of the participants were taking prophylactic medications for AMS, and none were taking medication known to increase HRV (beta-adrenoreceptor antagonists, statins, and angiotensin-converting enzyme inhibitors; Bilchick and Berger 2006). All participants gave written informed consent to participate and the study was approved by the National Institute for Social Care and Health Research Wales Research Ethics Service (14/WA/1260).

\section{Study design}

Baseline data were collected in the laboratories of Bangor University, North Wales (65 m above sea level). One month later participants completed an expedition that trekked on foot to a field laboratory at $3841 \mathrm{~m}$ and on to a maximum altitude of $5085 \mathrm{~m}$ (Manaslu circuit in the Nepali Himalaya). Participants were divided into five groups in which ascent profiles were essentially similar (Figure 1).

\section{Baseline data collection:}

To reduce variability in HRV measures, the participants were required to avoid drinking caffeine, alcohol or smoking for at least 12 hours, and to avoid eating and drinking for at least one hour before the measurements. Participants presented at the laboratory and were fitted with a commercially available heart rate monitor capable of obtaining HRV data (RS800CX, Polar Electro, Warwick, UK) and a peripheral oxygen saturation monitor (Onyx II 9550, Nonin, Minnesota, USA). Participants first lay supine in normoxia for 15 minutes with their eyes open and were asked to breathe normally and regularly. Paced breathing was not required of participants because it was 
deemed impractical for clinical settings and is not required to obtain reliable HRV data (Kox et al., 2011, Kobayashi, 2009). HRV data were collected for the last five minutes as previously recommended (Camm et al., 1996) and $\mathrm{SpO}_{2}$ was obtained in the final 30 s. Participants were then taken into a hypoxic chamber set at a $\mathrm{FiO}_{2}$ of $11.5 \%$ (equivalent to $5000 \mathrm{~m}$ ) for 15 minutes where the protocol above was repeated.

\section{Data collection during ascent:}

Under the supervision of each trekking group's research lead, participants kept a daily diary of various parameters including self-reported AMS symptoms by Lake Louise Score and $\mathrm{SpO}_{2}$ by fingertip pulse oximetry (as above). The same time of day, setting (for control of background ambient light) and finger was used each day for the measurement of $\mathrm{SpO}_{2}$ and each participant had a partner who obtained readings to ensure participants remained blinded to their results.

Participants had warm hands and obtained measures following overnight fast (Luks and Swenson, 2011). Preceding the overnight fast ( 8 hours), normal habitual caffeine intake was allowed.

In addition, at $3841 \mathrm{~m}$ the $\mathrm{HRV}$ and $\mathrm{SpO}_{2}$ were again obtained using the same data collection methods as at baseline but whilst breathing ambient air only.

\section{Data analysis:}

Data processing was performed by Polar Precision Performance software (version 3.02.007) and Kubios software (version 2.2) (Tarvainen et al.,2013) An initial examination of the HRV data excluded participants from analyses if their data were not considered physiologically plausible, or if the tachogram presented evident artifacts exceeding $5 \%$ of the total R-R intervals (Dos Santosa et al.,2013) (Figure 2). A low-level correction was then applied if the R-R Interval was lower than 0.35 seconds (refractory time), or R-R intervals that differed more than $20 \%$ from the preceding data point were detected. If the low-level correction detected more than $5 \%$ artifact in the HRV recording 
then the tachogram was also excluded from analyses (Lippman et al., 1994, Liu et al., 2013, Dos Santos et al., 2013, Wessel et al., 2000).

The power spectra were quantified by measuring the area in two frequency bands over a 5-min epoch. The LF power was calculated for frequency band $0.04-0.15 \mathrm{~Hz}$ and the HF power for frequency band $0.15-0.40 \mathrm{~Hz}$. The ratio of LF power to HF power (LF/HF) was also determined.

$\mathrm{SpO}_{2}$ data were expressed as the drop in $\mathrm{SpO}_{2}(\%)$ following exposure to hypoxia. AMS was diagnosed when participants were above $2500 \mathrm{~m}$ and had presence of headache and at least one other symptom by Lake Louise Score. These participants were classified as AMS susceptible. Participants not meeting these criteria were classified as AMS resistant. Total symptom score was also presented.

\section{Statistical analysis:}

Diagnostic accuracy analysis was performed to assess the ability of $\mathrm{HRV}$ and $\mathrm{SpO}_{2}$ to predict AMS susceptibility during the consequent exposure to altitude. Receiver operating characteristic (ROC) curve analysis to predict AMS susceptibility was applied to the HRV data obtained in three different environmental conditions: normobaric normoxia and normobaric hypoxia (both obtained at baseline) and hypobaric hypoxia (obtained during the trekking ascent at $3841 \mathrm{~m}$ ). Moreover, ROC curve analysis to predict AMS susceptibility was also applied to the drop in $\mathrm{SpO}_{2}$ between normoxia and normobaric hypoxia (after 15 minutes, $\mathrm{FiO}_{2}=11.5 \%$ ) and the drop in $\mathrm{SpO}_{2}$ between normoxia and hypobaric hypoxia (after 12 trekking days, $3841 \mathrm{~m}$ ). All the diagnostic accuracy analyses were performed using R 3.2.5 (R Foundation, www.r-project.org). OptimalCutpoint package was installed in R and it was used to obtain the area under ROC curve (AUC), the optimal cutpoint, the sensitivity, the specificity and the ROC curve plots (López-Ratón and Rodríguez-Álvarez, 2014). Epi and pROC packages were also installed in R and used to confirm the results concerning AUC generated by the 
OptimalCutpoint package. As results were the same only data from the OptimalCutpoint package is presented herein. The pROC package was also used to calculate the confidence intervals $(\mathrm{Cl})$ of the AUC. An AUC greater than 0.7 was considered fair; an AUC greater than 0.8 was considered moderate (Obuchowski et al., 2004); in both cases it was required that the $95 \% \mathrm{Cl}$ was greater than 0.5 (www.r-project.org). 


\section{Results:}

Of the 44 participants recruited at the beginning of the study, 28 provided acceptable $\mathrm{HRV}, \mathrm{SpO}_{2}$ and AMS data at all required time points. Therefore, only the data regarding these participants has been used for the data analysis and statistical analysis. The flow of participants through the study is shown in Figure 2. The mean $\mathrm{HRV}$ and $\mathrm{SpO}_{2}$ response to the various environmental conditions is shown in table 2 and the individual responses for selected variables is shown in Figure 3.

During the expedition 20 of the 28 participants showed AMS symptoms, giving an AMS incidence of 71.4\%. The length of time with AMS was mean (SD) 3.85 (2.23) days. Participants who presented with clinically defined AMS reported a total accumulative symptom score of 30.3 (14.5); participants who didn't present with clinically defined AMS reported a total accumulative symptom score of 12.3 (6.4).

Of the nine HRV predictor variables considered in this study, three variables presented fair diagnostic accuracy to predict AMS susceptibility (normoxia LF, chronic hypobaric hypoxia ratio and chronic hypobaric hypoxia LF) and two presented moderate diagnostic accuracy (normoxia ratio and acute normobaric hypoxia LF) (table 3). Of these two moderately accurate predictors, the normoxia ratio had the best diagnostic accuracy, having a higher AUC $(0.88$ vs. 0.80$)$ and better sensitivity and specificity, correctly identifying $85 \%$ of true positives (AMS susceptible participants) and $88 \%$ of true negatives (AMS resistant participants) (figure 4). Notably, LF had better diagnostic accuracy than HF in all environmental conditions, having AUC values greater than 0.7 (AUC of the HF variables were all lower than 0.7). Moreover, LF showed similar or better sensitivity and specificity than HF. In contrast, $\mathrm{SpO}_{2}$ variables considered in this study showed a poor diagnostic accuracy (AUC $\left.<0.7\right)$ with poor values of sensitivity and specificity (table 4 and figure 4). 


\section{Discussion:}

The aim of this study was to assess the diagnostic accuracy of pre- and intra-expedition HRV and $\mathrm{SpO}_{2}$ measurements to predict AMS susceptibility at high altitude.

The study's main findings were that LF/HF ratio in normoxia and LF following 15 minutes of exposure to normobaric hypoxia are promising variables to predict AMS during subsequent altitude exposure, showing moderate diagnostic accuracy (AUC greater than 0.8). Surprisingly, LF/HF ratio in normoxia had the best diagnostic accuracy (having the highest AUC), and the best sensitivity (85\%) and specificity (88\%) of all predictors. Three other variables had fair diagnostic accuracy (AUC greater than 0.7$)$ to predict AMS susceptibility: normoxia LF, chronic hypobaric hypoxia ratio and chronic hypobaric hypoxia LF. Generally, LF band HRV had better diagnostic accuracy than HF bands. In contrast, fall in $\mathrm{SpO}_{2}$ following 15 minutes of exposure to normobaric hypoxia or following 12 days exposure to hypobaric hypoxia had poor diagnostic accuracy (AUC less than 0.7).

We further found that at rest in normobaric normoxia, the mean LF/HF ratio was higher in the group of participants who did not subsequently develop AMS than in those who developed AMS. Despite this finding and the generally better diagnostic accuracy for this parameter compared to change in $\mathrm{SpO}_{2}$, scatterplots of individual data reveal there is still significant overlap of data points for $\mathrm{LF} / \mathrm{HF}$ ratio between the AMS and non-AMS groups (Figure 3).

\section{$\underline{\text { Heart Rate Variability }}$}

The relationship between hypoxia and HRV is complex, and thus it is unsurprising that there are inconsistent results in the literature (Loeppky et al., 2003; Lanfranchi et al., 2005; Chen et al., 2008; Huang et al., 2010; Karinen et al., 2012; Koehle et al., 2010; Wille et al., 2012; Mairer et al., 2013). Generally, hypoxia is thought to potently stimulate the sympathetic nervous system, causing a reduction of HRV variability (LF and HF components) and an increase in the LF/HF ratio (Zhang et al., 
2014). The results reported herein are consistent with this commonly observed response to hypoxia, showing a reduction in heart rate variability upon acute exposure that was somewhat normalised with chronic exposure.

The present study extends previous findings by identifying that an LF/HF ratio of less than 2.28 a.u. in normoxia could identify with reasonable diagnostic accuracy AMS-susceptibility. The only other study to have described risks of developing AMS by using a HRV cutoff is by Huang et al. (2010), who found a 7-fold increase in the odds of developing AMS in participants with an LF/HF ratio greater than 1.3 a.u. measured at $1317 \mathrm{~m}$. Although the present results do not concur with this finding, the measurements in Huang et al.'s study were taken in field conditions and were used to predict AMS at moderate altitude $(3440 \mathrm{~m})$. In contrast the present data were taken pre-expedition and were used to predict AMS at a much higher altitude $(5085 \mathrm{~m})$. Comparisons between the present and previous studies are also difficult because of differences in data analysis. For example, to our knowledge, this is the first study to have employed strict a priori-agreed exclusion criteria regarding implausible data and artefact, and the first study to include a diagnostic accuracy analysis to determine performance of HRV data obtained pre-expedition to predict AMS susceptibility.

In the present study, LF/HF ratio in normoxia had the best diagnostic accuracy, whilst the LF band demonstrated better diagnostic accuracy than $\mathrm{HF}$ under all conditions. As the mechanisms underlying HRV are complex, the exact physiological explanation of these findings are unclear. The traditional understanding that LF and HF components reflect sympathetic and parasympathetic activity, respectively, is now seen as overly simplistic (Scheff et al., 2014). It has recently been appreciated that other important factors in modulating HRV are mechanoreceptors and ventilatory response (Scheff et al., 2014), and that autonomic changes that affect heart rate tend to alter both LF and HF components (Chiu et al., 2003). Nevertheless it is tempting to speculate that since LF/HF ratio and LF take into account both sympathetic and parasympathetic cardiac supply, and may be related to baroreflex function, these HRV variables are better overall reflections of baroreflex 
activity, cardiac tone and sympatho-vagal balance (De Vito et al., 2002, Shaffer et al., 2014). Hence their performance to predict AMS susceptibility may be superior because they reflect the interaction of multiple physiological factors that cause AMS.

A surprising finding was the superior diagnostic accuracy of measurements taken in normoxia compared to hypoxia. Many studies have considered pre-expedition measurements in hypoxia to be a proxy for hypoxic conditions at true altitude, but it may be that individuals' physiological response to prolonged hypoxia, when given time to acclimatize, is different to acute hypoxic exposure. In this regard Zhang et al. (2014) found a significant increase in cardiorespiratory phase synchronization on exposure to acute hypoxia, which was associated with a lower HRV. Thus, variable ventilatory responses to acute hypoxia may confound the measurements of HRV if also obtained in acute hypoxia.

A further problem when comparing studies is that models of the effect of altitude on the autonomic nervous system often only consider the hypoxia variable, although both temperature and barometric pressure may have an independent effect on physiology at altitude (Conkin and Wessel, 2008; Coppel et al., 2015). Moreover, AMS symptoms can be less severe in normobaric hypoxia (Roach et al., 1996). Interestingly, the present finding that the best predictor for subsequent AMS is LF/HF ratio in normobaric normoxia suggests there are physiological differences between AMS-susceptible and AMS- resistant individuals that are observable even at sea level. The protective nature of a high HRV and a sympathovagal balance shifted to sympathetic dominance may be protective per se, or may reflect some other parameter, such as physical fitness or autonomic activity responsiveness, in particular to stressors such as hypoxia. Other 'sea level' physiological differences have been demonstrated previously, such as gag reflex (Austin and Sleigh, 1995) and resting blood pressure (Lanfranchi et al., 2005). In support of our findings, Akselrod et al. (2001) found that individuals with higher baseline autonomic activity were more resistant to desaturation on exposure to acute hypoxia. Although a recent study by Botek et al. (2015) found no correlation between the LF/HF ratio 
and delta $\mathrm{SpO}_{2}$, it was not determined whether $\mathrm{LF} / \mathrm{HF}$ ratio or delta $\mathrm{SpO}_{2}$ predicted subsequent AMS, so the lack of correlation may reveal the poor predictive value and the variable nature of the $\mathrm{SpO}_{2}$ response to acute hypoxia (rather than invalidating HRV measurements for field use).

\section{$\underline{\text { Oxygen saturations }}$}

Measurement of $\mathrm{SpO}_{2}$ at rest has previously been proposed as an indicator for inadequate acclimatization (Karinen et al., 2010), with several studies finding an inverse relationship between $\mathrm{SpO}_{2}$ and AMS-susceptibility (Mairer et al., 2013, Karinen et al., 2010). More severe desaturation in hypoxia in AMS-susceptible participants is probably due to differences in ventilatory response (Burtscher et al., 2004) or a ventilation/perfusion mismatch (Loeppky et al, 2008). However, measurement of $\mathrm{SpO}_{2}$ for 15 minutes in acute normobaric hypoxia and chronic hypobaric hypoxia in the present study showed poor diagnostic accuracy. Indeed, other studies have found no association (Wille et al., 2012). Although some authors have proposed that inconsistencies may be due to differences in the time of measurement $\left(\mathrm{SpO}_{2}\right.$ measured after $>20$ minutes in hypoxia, but within the first hour, appears to have superior predictive value than later measurements (Burtscher et al., 2008 , Loeppky et al., 2008)), it is an unlikely explanation in the present study because data were also taken after chronic exposure to hypobaric hypoxia, and still showed poor diagnostic accuracy. It is conceivable that the peripheral oxygen saturation method may be unreliable, even though the present methodology was standardised and likely to be more reliable than if used outside of the research setting.

\section{Strengths and limitations of the study}

The rate of ascent and previous acclimatization can affect the likelihood of developing AMS (Bartsch and Saltin, 2008). The ascent profiles for all participant groups were similar and all participants climbed the same route (Figure 1). None of the participants had been to altitude within the month proceeding the expedition. All data was collected at the same time after reaching a given altitude 
with control of variables such as caffeine, smoking and eating prior to collection of HRV data. We also gave advice to ensure participants stayed well hydrated during the expedition. Thus, many of the variables that can influence AMS and HRV were well controlled (enhancing internal validity). We note that uncontrolled variables such as age and sex can influence HRV, however the outcome measure of AMS was obtained in the field in a group of heterogeneous participants representative of typical high altitude sojourners (enhancing external validity). We have also provided detailed explanation of the criterion used to exclude HRV data due to artefact or physiologically implausible data. Combined with the use of diagnostic accuracy statistics and individual data plots, the study thus allows a meaningful interpretation of the utility of $\mathrm{HRV}$ at different time points (before or during the expedition) to predict AMS.

A major concern regarding use of HRV to predict AMS susceptibility is that of 35 complete data sets, 6 participants' data had to be excluded due to artefacts or implausible data (Figure 2), despite controls such as lying still and not talking. Thus, until the data collection equipment is improved or simplified, there will be participants for whom prediction of AMS susceptibility is currently not possible. It is also possible that HRV simply reflects some other unidentified predictor of AMS (but exploratory analyses of other independent variables could not identify any such predictor).

Finally, it is well known that respiratory rate effects heart rate variability and peripheral oxygen saturations. Bilman (2011) discussed the effect of respiratory parameters on heart rate variability, suggesting that both respiratory rate and tidal volume alter heart rate variability, including both the HF and LF components. Lehrer and Gevirtz (Lehrer and Gevirtz, 2014) suggest that a respiratory rate between 4.5 and 7 breaths per minute improves autonomic homeostasis via heart rate variability biofeedback. They further suggest that breathing at the rate of the cardiovascular systems resonance frequency can enhance performance. However, respiratory rate is grossly effected by altitude (Richard et al., 2014); controlling and thus changing a participant's respiratory rate would not reflect their physiological state during the rest of the expedition. Furthermore, it is practical to 
control respiratory rate in normobaric normoxia, but may be impossible to control respiratory rate in normobaric or hypobaric hypoxia. Instead, we told participants to lie awake and breath normally, thus avoiding periodic breathing. As for peripheral oxygen saturations, some variability in this measure will have arisen due to using only acute exposure to normobaric hypoxia. Such variability may lead to an underestimation of the diagnostic accuracy of $\mathrm{SpO}_{2}$. However, previous observations by our group suggest this variability is considerably reduced at 15 minutes compared to initial exposure. Thus for both heart rate variability and peripheral oxygen saturation we chose to make a compromise between scientific rigor and practically implementable methods that would most likely be adopted in the field.

\section{Implications for practice}

In the present study, an accurate predictor for AMS susceptibility was identified using a simple tool implementable at sea level. This is important, because for a diagnostic tool for AMS to be clinically useful, results must be available in time to make adjustments to prevent disease. For example, lengthening the ascent profile, or taking prophylactic mediation. A recent study by Karinen et al. (2012) found root mean square differences of HRV at $2400 \mathrm{~m}$ had $92 \%$ sensitivity for detecting AMS if ascent continued to $3000-4300 m$ without acclimatization. However, the need for a field laboratory and technicians at $2400 \mathrm{~m}$, at which point some individuals may already be suffering from AMS (Burtscher et al., 2008) and from which altitude it may be too late to change itinerary, make these measurements unwieldy and unsuitable as methods of predicting disease (Song et al., 2013). Even studies finding potentially clinically useful pre-expedition predictors usually require long exposure to simulated altitude (Mairer et al., 2013), which is demanding on time, technology, and finances.

LF/HF ratio of less than 2.28 in normoxia was recognized as being the best cutpoint to identify individuals at risk of AMS during altitude exposure. This has interesting practical implications because it suggests that hypoxic exposure pre-expedition may not be required to predict AMS 
susceptibility. This finding is consistent with the results of Rossetti et al. (2016; in press), who found that normoxic $\mathrm{VO}_{2}$ max predicts altitude performance without measures of hypoxic sensitivity. Our findings also give an insight into cardiac autonomic responses in regard to AMS development and might help to clarify the question of whether HRV modulations appear as a consequence and/or cause of AMS.

Currently, however, post-processing of the HRV data is still required, making interpretations of the results difficult without some technical knowledge. Also until equipment is improved, any HRV test should last at least 10 minutes, to allow for removal of artefact from the recording. Modification of existing heart rate monitors should be considered, to give information to users pre-expedition on their susceptibility to AMS at true altitude, by measuring LF/HF ratio in normoxia. A simple diagnostic test incorporated into a watch has the potential to reduce AMS by allowing implementation of preventative measures, but should be used with caution to prevent participants who are predicted to be AMS resistant from ignoring general advice on AMS prevention. Finally, at present some overlap in HRV responses between AMS susceptible and AMS resistant individuals exists. Therefore, further research is needed to validate our findings with an increased number of more heterogeneous participants, of varying degrees of AMS, to determine the true utility of HRV data to predict AMS.

\section{Conclusion}

This study showed that HRV has better diagnostic accuracy for AMS prediction than peripheral $\mathrm{SpO}_{2}$ saturation monitoring. These results show the measurement with the highest diagnostic accuracy is normoxic LF/HF ratio, measured pre-expedition. Further confirmatory studies are needed to validate these measurements, but they suggest an alternative approach to obtain a more accurate, simple method of predicting AMS. 


\section{Author Disclosure Statement}

The authors declare no competing interests.

\section{Acknowledgements}

The authors would like to thank all research participants who took part in the MEDEX 2015 expedition. 


\section{References:}

Akselrod S, Barak Y, Ben-Dov Y, Keselbrener, L and Baharav A (2001). Estimation of autonomic response based on individually determined time axis. Auton Neurosci, 90, 13-23.

Austin D and Sleigh J (1995). Prediction of acute mountain sickness. The BMJ, 311, 989-90.

Bartsch P and Saltin B (2008). General introduction to altitude adaptation and mountain sickness. Scand J Med Sci Sports, 18 Suppl 1, 1-10.

Billman GE (2011). Heart rate variability - a historical perspective. Front Physiol, 2, 86.

Billman GE (2013). The LF/HF ratio does not accurately measure cardiac sympatho-vagal balance. Front Physiol, 4.

Botek M, Krejci J, De Smet S, Gaba A and Mckune AJ (2015). Heart rate variability and arterial oxygen saturation response during extreme normobaric hypoxia. Auton Neurosci, 190, 40-5.

Brierley G, Parks T and Wolff CB (2012). The Relationship of Acute Mountain Sickness to Arterial Oxygen Saturation at Altitudes of 3324 to 5176 m. In: Wolf M, Bucher HU, Rudin M, Van Huffel S, Wolf U, Bruley DF, Harrison DK (eds). Oxygen Transport to Tissue XXXIII. New York NY: Springer New York

Burtscher M, Flatz M and Faulhaber M (2004). Prediction of susceptibility to acute mountain sickness by $\mathrm{SaO} 2$ values during short-term exposure to hypoxia. High Alt Med Biol 5:335-40

Burtscher M, Szubski C and Faulhaber M (2008). Prediction of the susceptibility to AMS in simulated altitude. Sleep Breath 12:103-8

Camm AJ, Malik M and Bigger JT (1996) Heart rate variability: standards of measurement physiological interpretation and clinical use. Task Force of the European Society of Cardiology and the North American Society of Pacing and Electrophysiology Circulation. 93

Chalmers JA, Quintana DS, Abbott MJ, and Kemp AH (2014). Anxiety Disorders are Associated with Reduced Heart Rate Variability: A Meta-Analysis. Front Psychiatry 5:80

Chen YC, Lin FC, Shiao GM, and Chang SC (2008). Effect of rapid ascent to high altitude on autonomic cardiovascular modulation. Am J Med Sci 336:248-53

Chiu HW, Wang TH, Huang LC, Tso HW, and Kao T (2003). The influence of mean heart rate on measures of heart rate variability as markers of autonomic function: a model study. Med Eng Phys 25:475-81

Cohen MA and Taylor JA (2002). Short-term cardiovascular oscillations in man: measuring and modelling the physiologies. J Physiol 542:669-83

Conkin J and Wessel JH (2008). Critique of the equivalent air altitude model. Aviat Space Environ Med 79:975-82

Coppel J, Hennis P, Gilbert-Kawai E, and Grocott MP (2015). The physiological effects of hypobaric hypoxia versus normobaric hypoxia: a systematic review of crossover trials. Extrem Physiol Med 4:2

De Vito G, Galloway SD, Nimmo MA, Maas P, and Mcmurray JJ (2002). Effects of central sympathetic inhibition on heart rate variability during steady-state exercise in healthy humans. Clin Physiol Funct Imaging 22:32-8

Dos Santos L, Barroso JJ, Macau EE, and De Godoy MF (2013) Application of an automatic adaptive filter for Heart Rate Variability analysis. Med Eng Phys 35:1778-85

Duplain H, Vollenweider L, Delabays A, Nicod P, Bartsch P, and Scherrer U (1999). Augmented sympathetic activation during short-term hypoxia and high-altitude exposure in subjects susceptible to high-altitude pulmonary edema. Circulation 99:1713-8

Hackett PH (1999). The cerebral etiology of high-altitude cerebral edema and acute mountain sicknes.s Wilderness Environ Med 10:97-109 
Honigman B, Theis MK, Koziol-Mclain J, Roach R, Yip R, Houston C, Moore LG, and Pearce P (1993). Acute mountain sickness in a general tourist population at moderate altitudes. Ann Intern Med 118:587-92

Huang HH, Tseng CY, Fan JS, Yen DHT, Kao WF, Chang SC, Kuo TBJ, Huang Cl, and Lee C H (2010). Alternations of heart rate variability at lower altitude in the predication of trekkers with acute mountain sickness at high altitude Clin J Sport Med 20

Kanai M Nishihara F Shiga T Shimada H \& Saito S 2001 Alterations in autonomic nervous control of heart rate among tourists at 2700 and $3700 \mathrm{~m}$ above sea level. Wilderness Environ Med 12 8-12

Karinen HM, Peltonen JE, Kahonen M, and Tikkanen HO (2010). Prediction of acute mountain sickness by monitoring arterial oxygen saturation during ascent High Alt Med Biol 11:325-32

Karinen HM, Uusitalo A, Vaha-Ypya H, Kahonen M, Peltonen JE, Stein PK, Viik J, and Tikkanen HO (2012). Heart rate variability changes at $2400 \mathrm{~m}$ altitude predicts acute mountain sickness on further ascent at 3000-4300 m altitudes. Front Physiol 3:336

Kobayashi H (2009) Does paced breathing improve the reproducibility of heart rate variability measurements? J Physiol Anthropol 28:225-30

Koehle MS, Guenette JA, and Warburton DE (2010) Oximetry heart rate variability and the diagnosis of mild-to-moderate acute mountain sickness. Eur J Emerg Med 17

Kox M, Pompe JC, Van Der Hoeven JG, Hoedemaekers CW, and Pickkers P (2011). Influence of different breathing patterns on heart rate variability indices and reproducibility during experimental endotoxaemia in human subjects. Clin Sci 121:215-22

La Rovere MT, Bigger JT Jr, Marcus FI, Mortara A, and Schwartz PJ (1998). Baroreflex sensitivity and heart-rate variability in prediction of total cardiac mortality after myocardial infarction ATRAMI (Autonomic Tone and Reflexes After Myocardial Infarction). Investigators Lancet 351:478-84

Lake Louise Consensus on the Definition and Quantification of Altitude Illness (1991). Hypox Mount Med. Proceedings of the 7th International Hypoxia Symposium, Lake Louise Canada February

Lanfranchi PA, Colombo R, Cremona G, Baderna P, Spagnolatti L, Mazzuero G, Wagner P, Perini L, Wagner H, Cavallaro C, and Giannuzzi P (2005) Autonomic cardiovascular regulation in subjects with acute mountain sickness. Am J Physiol Heart Circ Physiol 289:H2364-72

Lehrer PM, and Gevirtz R (2014). Heart rate variability biofeedback: how and why does it work? Frontiers in Psychology 5

Lippman N, Stein KM, and Lerman BB (1994). Comparison of methods for removal of ectopy in measurement of heart rate variability. American Journal of Physiology - Heart and Circulatory Physiology 267:H411-H418

Liu NT, Batchinsky AI, Cancio LC, and Salinas J (2013). The impact of noise on the reliability of heartrate variability and complexity analysis in trauma patients. Comput Biol Med 43:1955-64

Loeppky JA, Icenogle MV, Charlton G, Conn CA, Maes D, Riboni K, Gates L, Melo MF, and Roach RC (2008). Hypoxemia and acute mountain sickness: which comes first? High Alt Med Biol 9:271-9

Loeppky JA, Icenogle MV, Maes D, Riboni K, Scotto P, and Roach R C (2003). Body temperature autonomic responses and acute mountain sickness. High Alt Med Biol 4 367-73

López-Ratón M, Rodríguez-Álvarez MX. OptimalCutpoints: computing optimal cutpoints in diagnostic tests R package version 11-3 2014 Available at: http://CRANRprojectorg/package=OptimalCutpoints [Accessed 19 ${ }^{\text {th }}$ November 2016]

Luks AM and Swenson ER (2011). Pulse oximetry at high altitude. High Alt Med Biol 12:109-19

Maggiorini M, Bühler B, Walter M, and Oelz O (1990). Prevalence of acute mountain sickness in the Swiss Alps Bmj 301:853-5

Mairer K, Wille M, Grander W, and Burtscher M (2013). Effects of exercise and hypoxia on heart rate variability and acute mountain sickness. Int J Sports Med 34:700-6 
Malliani A, Pagani M, Lombardi F, and Cerutti S (1991). Cardiovascular neural regulation explored in the frequency domain. Circulation 84:482-492

Obuchowski NA, Lieber ML, and Wians FH Jr (2004). ROC curves in clinical chemistry: uses misuses and possible solutions. Clin Chem 50:1118-25

Oliver SJ, Sanders SJ, Williams CJ, Smith ZA, Lloyd-Davies E, Roberts R, Arthur C, Hardy L, and Macdonald JH (2012). Physiological and psychological illness symptoms at high altitude and their relationship with acute mountain sickness: a prospective cohort study. J Travel Med 19:210-9

Rahman F, Pechnik S, Gross D, Sewell L, and Goldstein DS (2011). Low frequency power of heart rate variability reflects baroreflex function not cardiac sympathetic innervation Clin Auton Res 21:133-41

Richard NA, Sahota IS, Widmer N, Ferguson S, Sheel AW, and Koehle MS (2014). Acute mountain sickness chemosensitivity and cardiorespiratory responses in humans exposed to hypobaric and normobaric hypoxia. Journal of Applied Physiology 116:945-952

Roach RC, Loeppky JA, and Icenogle MV (1996). Acute mountain sickness: increased severity during simulated altitude compared with normobaric hypoxia. J Appl Physiol 81:1908-10

Rossetti GMK, Macdonald JH, Smith M, Jackson AR, Callender N, Newcombe HK, Storey HM, Willis S, van den Beukel J, Woodward J, Pollard J, Wood B, Newton V, Virian J, Haswell O, and Oliver SJ. MEDEX2015: Greater sea-level fitness is associated with lower sense of effort during Himalayan trekking without worse Acute Mountain Sickness. High Alt Med Biol; In Press.

Scheff JD, Griffel B, Corbett SA, Calvano SE, and Androulakis IP (2014). On heart rate variability and autonomic activity in homeostasis and in systemic inflammation. Math Biosci 252:36-44

Shaffer F, Mccraty R, and Zerr CL (2014). A healthy heart is not a metronome: an integrative review of the heart's anatomy and heart rate variability. Front Psychol 5

Song H, Ke T, Luo W-J, and Chen J-Y (2013). Non-high altitude methods for rapid screening of susceptibility to acute mountain sickness. BMC Public Health 13:902

Task Force of the European Society of Cardiology and the North American Society of Pacing and Electrophysiology. (1996) Heart rate variability Standards of measurement physiological interpretation and clinical use. Eur Heart J 17:354-81

Wessel N, Voss A, Malberg H, Ziehmann C, Voss HU, Schirdewan A, Meyerfeldt U, and Kurths J (2000). Nonlinear analysis of complex phenomena in cardiological data. Herzschrittmacherther und Elektrophysiol 11:159-173

Wille M, Mairer K, Gatterer H, Philippe M, Faulhaber M, and Burtscher M (2012). Changes in cardiac autonomic activity during a passive 8 hour acute exposure to $5500 \mathrm{~m}$ normobaric hypoxia are not related to the development of acute mountain sickness. Int J Sports Med 33:186-91

Zhang D, She J, Zhang Z, and Yu M (2014). Effects of acute hypoxia on heart rate variability sample entropy and cardiorespiratory phase synchronization. BioMedical Engineering OnLine 13:73 
Table legends.

Table 1. Participant demographics.

\begin{tabular}{|c|c|c|c|c|}
\hline & \multicolumn{2}{|c|}{ Participants included: } & \multicolumn{2}{|c|}{ Participants excluded: } \\
\hline & Male & Female & Male & Female \\
\hline \multirow[t]{2}{*}{ Gender (n) } & 17 & 11 & 9 & 7 \\
\hline & Mean & SD & Mean & SD \\
\hline Age (y) & 34.0 & 12.7 & 42.9 & 13.4 \\
\hline Height $(\mathrm{cm})$ & 172 & 10 & 171 & 10 \\
\hline Body mass (Kg) & 66.3 & 12.0 & 70.9 & 13.6 \\
\hline $\mathrm{VO}_{2} \max (\mathrm{ml} / \mathrm{kg} / \mathrm{min})$ & 46.69 & 6.02 & 44.50 & 9.66 \\
\hline Living altitude (m) & 74 & 118 & 85 & 100 \\
\hline Days mountaineering $(\mathrm{n})$ & 9 & 12 & 5 & 9 \\
\hline \multirow[t]{2}{*}{ Physical activity score (a.u) } & 5.3 & 1.5 & 4.2 & 2.5 \\
\hline & \multicolumn{2}{|c|}{$\begin{array}{c}\text { Participants included: } \\
\text { Yes }\end{array}$} & \multicolumn{2}{|c|}{$\begin{array}{c}\text { Participants excluded: } \\
\text { Yes }\end{array}$} \\
\hline AMS susceptible on the expedition ( $n$ ) & \multicolumn{2}{|c|}{20} & \multicolumn{2}{|c|}{6} \\
\hline \multicolumn{4}{|l|}{ Previous experience of acute mountain } & \\
\hline \multicolumn{5}{|l|}{ Previous experience of high altitude } \\
\hline \multicolumn{4}{|l|}{ Previous experience of high altitude } & \\
\hline Smoker (n) & \multicolumn{2}{|c|}{1} & \multicolumn{2}{|c|}{1} \\
\hline Consumes alcohol ( $\mathrm{n}$ ) & \multicolumn{2}{|c|}{27} & \multicolumn{2}{|c|}{15} \\
\hline History of headache/migraine (n) & \multicolumn{2}{|c|}{3} & \multicolumn{2}{|c|}{5} \\
\hline Consumes dietary supplement & \multicolumn{2}{|c|}{2} & \multicolumn{2}{|c|}{3} \\
\hline Previous history of travel above $3000 \mathrm{~m}(\mathrm{n})$ & \multicolumn{2}{|c|}{26} & \multicolumn{2}{|c|}{15} \\
\hline
\end{tabular}


Table 2. Heart rate variability response and peripheral oxygen saturation response to hypoxic exposure assessed before and during a high altitude expedition. HRV heart rate variability; LF low frequency; $\mathrm{HF}$ high frequency; $\Delta \mathrm{SpO}_{2}$, drop in peripheral oxygen saturation between normoxia and hypoxia.

\begin{tabular}{|c|c|c|c|}
\hline Environmental condition & Variable & Mean & SD \\
\hline & HRV ratio (a.u.) & 1.99 & 1.35 \\
\hline After $15 \mathrm{~min}$ exposure to & HRV LF $\left(\mathrm{ms}^{2}\right)$ & 1380 & 1096 \\
\hline & $\mathrm{HRV} \mathrm{HF}\left(\mathrm{ms}^{2}\right)$ & 1088 & 1953 \\
\hline & HRV ratio (a.u.) & 3.91 & 3.13 \\
\hline After 15 min exposure to & HRV LF $\left(\mathrm{ms}^{2}\right)$ & 1031 & 845 \\
\hline \multirow[t]{3}{*}{ normobaric hypoxia } & $\mathrm{HRV} \mathrm{HF}\left(\mathrm{ms}^{2}\right)$ & 347 & 273 \\
\hline & $\Delta \mathrm{SpO}_{2}(\%)$ & 21.1 & 7.2 \\
\hline & HRV ratio (a.u.) & 2.78 & 3.44 \\
\hline After 12 days exposure to & HRV LF $\left(\mathrm{ms}^{2}\right)$ & 1246 & 1604 \\
\hline \multirow[t]{2}{*}{ hypobaric hypoxia } & $\mathrm{HRV} \mathrm{HF}\left(\mathrm{ms}^{2}\right)$ & 763 & 974 \\
\hline & $\Delta \mathrm{SpO}_{2}(\%)$ & 9.7 & 2.5 \\
\hline
\end{tabular}


Table 3. Diagnostic accuracy of heart rate variability assessed before and during a high altitude expedition to determine susceptibility to Acute Mountain Sickness. HRV heart rate variability; LF low frequency; HF high frequency; AUC (CI) area under receiver operating characteristic curve $(95 \%$ confidence interval).

\begin{tabular}{|c|c|c|c|c|c|}
\hline $\begin{array}{l}\text { Environmental } \\
\text { condition }\end{array}$ & $\begin{array}{c}\text { HRV } \\
\text { variable }\end{array}$ & $\mathbf{A U C}(\mathbf{C I})$ & $\begin{array}{c}\text { Sensitivity } \\
\%\end{array}$ & $\begin{array}{c}\text { Specificity } \\
\%\end{array}$ & $\begin{array}{l}\text { Optimal } \\
\text { cut-off }\end{array}$ \\
\hline After 15 min exposure & Ratio & $0.875(0.741-1,000)$ & 85 & 87.5 & $\leq 2.28$ a.u. \\
\hline \multirow{2}{*}{$\begin{array}{l}\text { to normobaric } \\
\text { normoxia }\end{array}$} & $\mathrm{LF}$ & $0.781(0.591-0.972)$ & 75 & 75 & $>552 \mathrm{~ms}^{2}$ \\
\hline & $\mathrm{HF}$ & $0.506(0.235-0.753)$ & 50 & 55 & $>1252 \mathrm{~ms}^{2}$ \\
\hline \multirow{3}{*}{$\begin{array}{l}\text { After } 15 \text { min exposure } \\
\text { to normobaric hypoxia }\end{array}$} & Ratio & $0.638(0.380-0.895)$ & 70 & 62.5 & $\leq 3.13$ a.u. \\
\hline & $\mathrm{LF}$ & $0.800(0.627-0.973)$ & 70 & 75 & $>275 \mathrm{~ms}^{2}$ \\
\hline & $\mathrm{HF}$ & $0.694(0.424-0.963)$ & 70 & 75 & $>740 \mathrm{~ms}^{2}$ \\
\hline After 12 days & Ratio & $0.731(0.532-0.931)$ & 70 & 75 & $\leq 1.91$ a.u. \\
\hline exposure to & $\mathrm{LF}$ & $0.725(0.503-0.947)$ & 80 & 62.5 & $>276 \mathrm{~ms}^{2}$ \\
\hline hypobaric hypoxia & $\mathrm{HF}$ & $0.625(0.383-0.867)$ & 60 & 62.5 & $>777 \mathrm{~ms}^{2}$ \\
\hline
\end{tabular}


Table 4. Diagnostic accuracy of peripheral oxygen desaturation assessed before and during a high altitude expedition to determine susceptibility to Acute Mountain Sickness. AUC (CI) area under receiver operating characteristic curve ( $95 \%$ confidence interval).

\begin{tabular}{lllll}
\hline Environmental & AUC $(\mathbf{C I})$ & Sensitivity & Specificity & Optimal cut-off \\
condition & & $\%$ & $\%$ & \\
\hline After 15 min exposure & $0.688(0.478-0.897)$ & 65 & 75 & $>21 \%$ \\
to normobaric hypoxia & & & & \\
After 12 days & & & 75 & $>10 \%$ \\
exposure to hypobaric & $0.688(0.449-0.926)$ & 65 & & \\
hypoxia & & & & \\
\hline
\end{tabular}




\section{Figure Legends.}

Figure 1: Ascent profile for all groups.

Figure 1.

..... Group 1 Group 2 - - Group $3 \longrightarrow$ Group 4 - Group 5

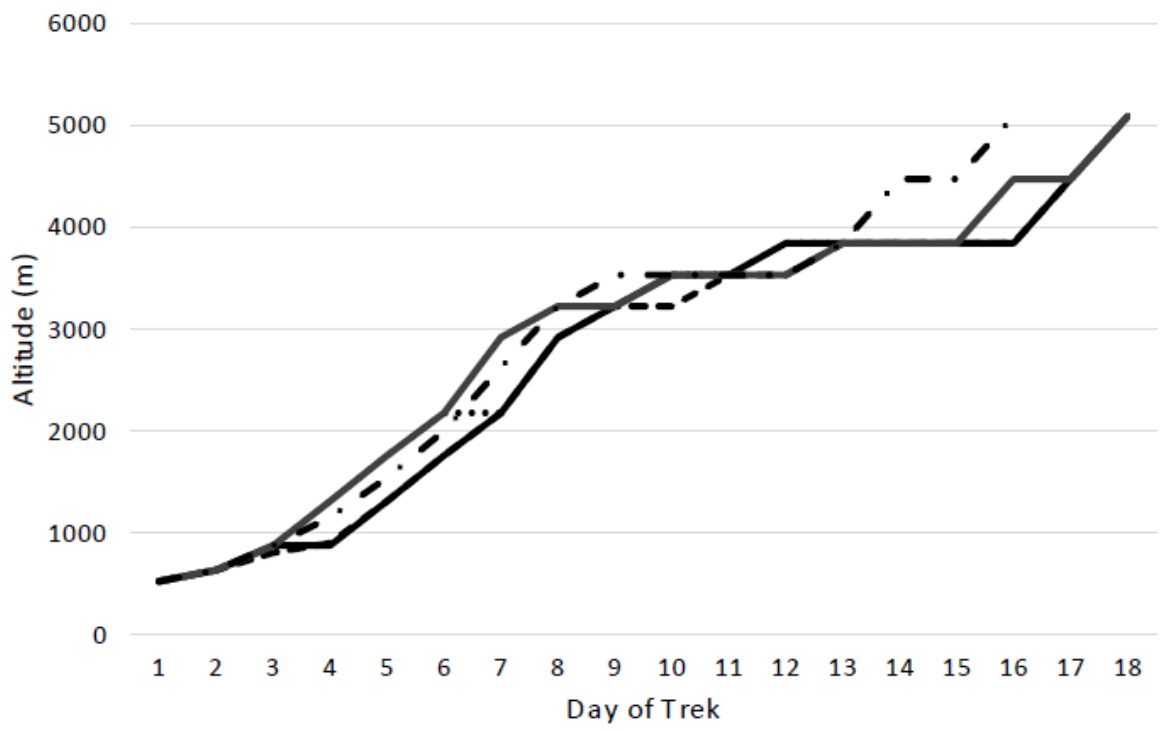


Figure 2. Participant flow through the study.

Figure 2.

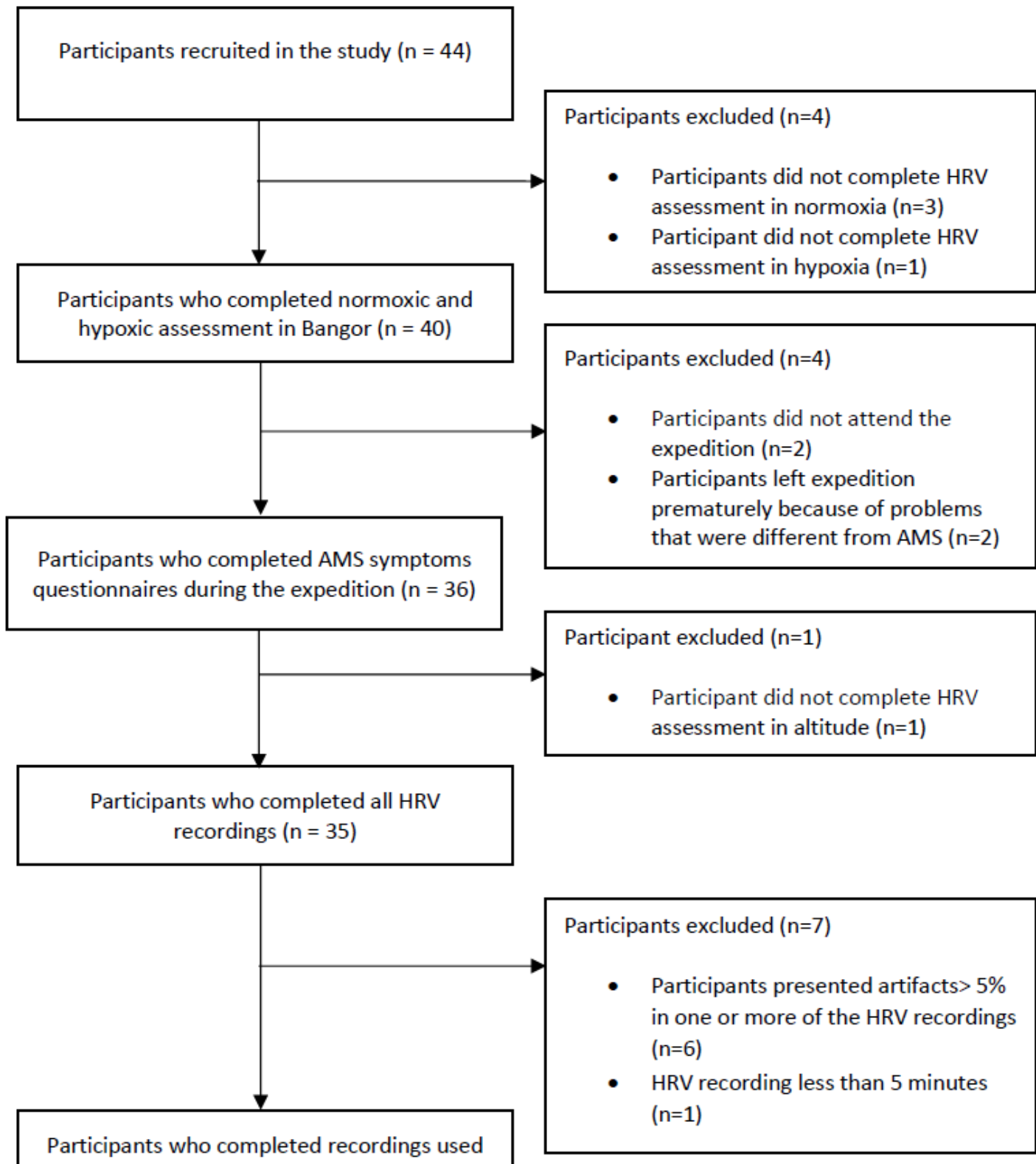

for the diagnostic accuracy analysis $(n=28)$ 
Figure 3: Individual responses to hypoxic exposure for selected $\mathrm{HRV}$ and $\mathrm{SpO}_{2}$ variables assessed before and during a high altitude expedition. HRV heart rate variability; LF low frequency; HF high frequency; $\Delta \mathrm{SpO}_{2}$ drop in peripheral oxygen saturation between normoxia and hypoxia

Normobaric-normoxia LF/HF ratio

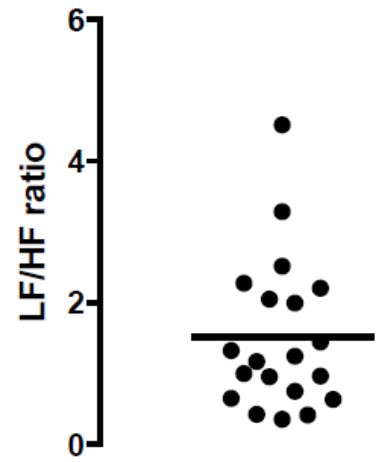

AMS

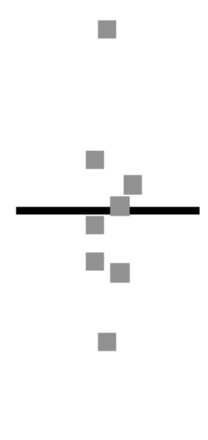

non AMS

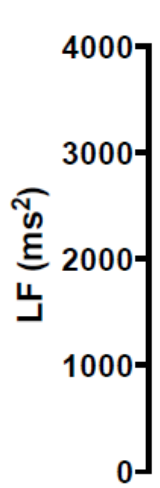

Normobaric-hypoxia LF

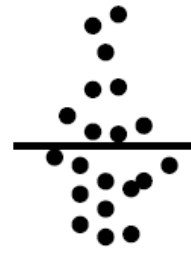

-

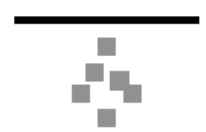

non AMS
Normobaric-hypoxia ${ }_{\Delta} \mathrm{SpO}_{2}$

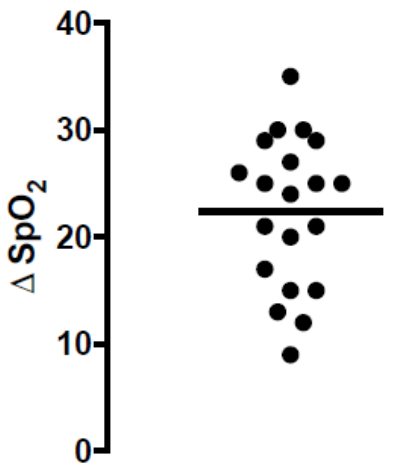

AMS

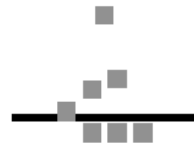

$\square$

non AMS
Hypobaric-hypoxia $\Delta \mathrm{SpO}_{2}$

$\left.\begin{array}{lll} & & 15 \\ & & \\ & & \\ & & \\ \text { के } & & \\ 4 & & \\ & & \\ & \\ & \\ & & \\ \end{array}\right]$

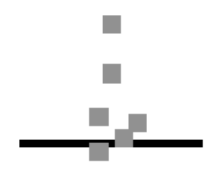

$\square$

$\square$

AMS

non AMS 
Figure 4: Receiver operating characteristic curve for selected heart rate variability and peripheral oxygen desaturation variables assessed before a high altitude expedition to determine susceptibility to Acute Mountain Sickness. LF low frequency; $\mathrm{SpO}_{2}$ drop in peripheral oxygen saturation between normoxia and normobaric hypoxia

Figure 4.

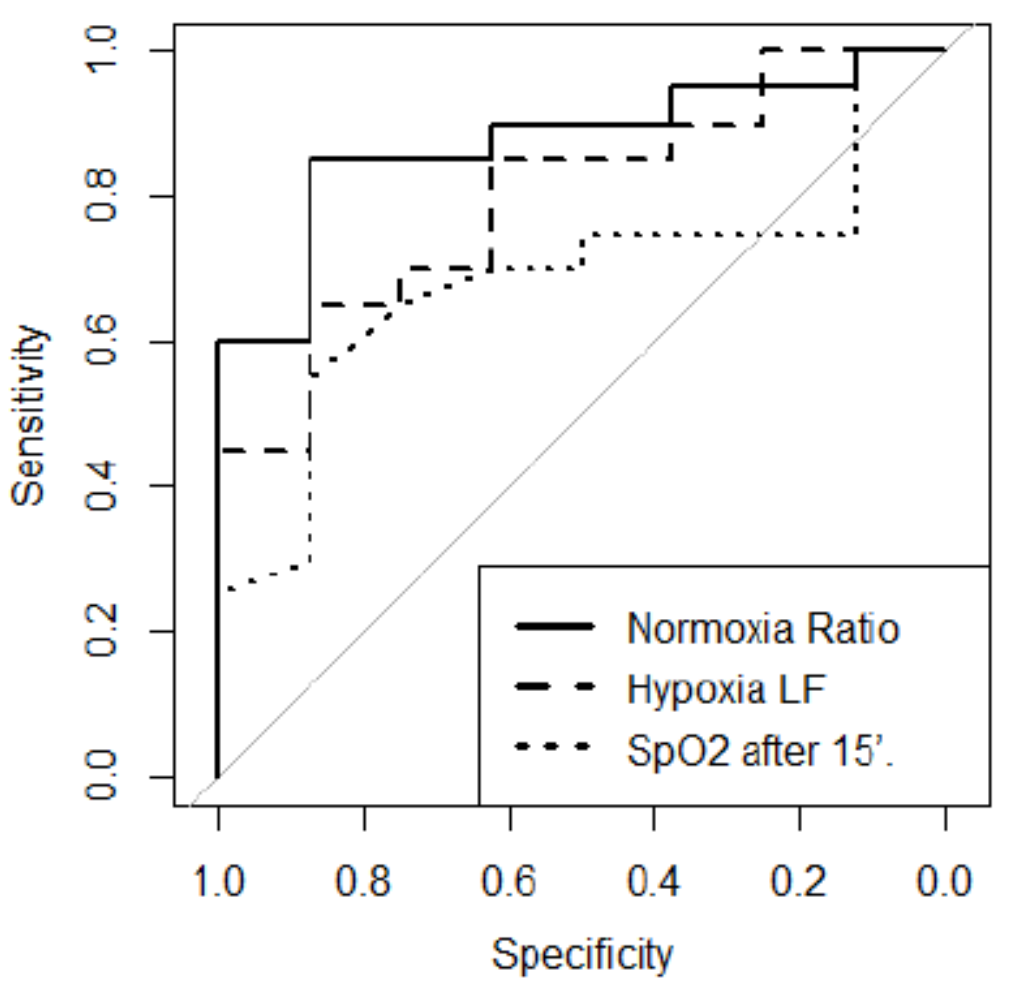

